\title{
Proceedings of the 2019 GRAPPA Collaborative Research Network Meeting
}

\author{
Robin Waxman, Carmel Stober (1D), Deepak R. Jadon, Ana-Maria Orbai (iD), Vinod Chandran (D), \\ Alexis Ogdie (iD), Stephen R. Pennington (1), Maarten de Wit ${ }^{\mathbb{D}}$, Denis O’Sullivan (D), \\ Philip J. Mease (D), April W. Armstrong (D), Kristina Callis Duffin (D), Philip S. Helliwell (D),

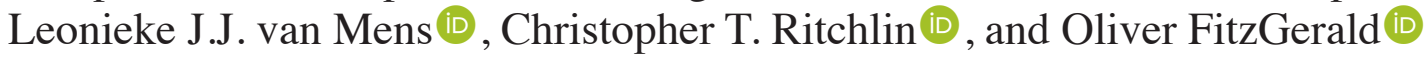

\begin{abstract}
At the 2019 Group for Research and Assessment of Psoriasis and Psoriatic Arthritis-Collaborative Research Network annual meeting, the group presented its progress in selecting a database platform; items to include in an electronic case report form (eCRF); and standardized operating procedures (SOP) for the collection, processing, storage, and transport of biomaterial. A pilot investigator-initiated study was also proposed that, in addition to addressing an area of unmet need, would allow for the testing of both the eCRF and SOP. (J Rheumatol Suppl. 2020 June;96:25-30; doi:10.3899/jrheum.200123)
\end{abstract}

Key Indexing Terms:

PSORIATIC ARTHRITIS PSORIASIS GRAPPA BIOMARKERS RESEARCH

From the School of Medicine, Leeds Institute of Rheumatic and Musculoskeletal Medicine, University of Leeds, Leeds; Cambridge University Hospitals National Health Service (NHS) Foundation Trust, Cambridge, UK; Division of Rheumatology, Johns Hopkins University School of Medicine, Baltimore, Maryland, USA; Department of Medicine, Division of Rheumatology, University of Toronto, Toronto Western Hospital, Krembil Research Institute, Toronto, Ontario, Canada; Perelman School of Medicine, University of Pennsylvania, Philadelphia, Pennsylvania, USA; Conway Institute for Biomolecular Research, University College Dublin, Dublin, Ireland; Patient Research Partner, Our Lady's Hospice and Care Services, Dublin, Ireland; Swedish Medical Center/Providence-St. Joseph Health and University of Washington, Seattle, Washington, USA; University of Southern California, Los Angeles, California; University of Utah, Salt Lake City, Utah, USA; Bradford Hospitals NHS Foundation Trust, Bradford, UK; Amsterdam University Medical Centers/University of Amsterdam, Department of Clinical Immunology and Rheumatology Amsterdam, Infection \& Immunity Institute, Amsterdam, the Netherlands; Division of Allergy, Immunology, and Rheumatology, University of Rochester Medical Center, Rochester, New York, USA; Department of Rheumatology, St. Vincent's University Hospital Dublin, Dublin, Ireland.

As part of the supplement series GRAPPA 2019, this report was reviewed internally and approved by the Guest Editors for integrity, accuracy, and consistency with scientific and ethical standards.

R. Waxman, MPH, Research Assistant, School of Medicine, Leeds Institute of Rheumatic and Musculoskeletal Medicine, University of Leeds; C. Stober, PhD, MBChB, MRCP, Consultant Rheumatologist, Cambridge University Hospitals NHS Foundation Trust; D.R. Jadon, MBBCh, MRCP, $P h D$, Director of Rheumatology Research Unit, Cambridge University Hospitals NHS Foundation Trust; A.M. Orbai, MD, MHS, Assistant Professor of Medicine, Director Psoriatic Arthritis Program, Division of Rheumatology, Johns Hopkins University School of Medicine;

$V$. Chandran, MBBS, MD, DM, PhD, Department of Medicine, Division of Rheumatology, University of Toronto, Toronto Western Hospital, Krembil Research Institute; A. Ogdie, MD, Assistant Professor of Medicine and Epidemiology, Perelman School of Medicine, University of Pennsylvania; S.R. Pennington, PhD, Professor of Proteomics, Conway Institute for Biomolecular Research, University College Dublin; M. de Wit, PhD, Patient Research Partner, Amsterdam; D. O'Sullivan, BE, Patient Research Partner, Our Lady's Hospice and Care Services; P.J.Mease, MD, Swedish Medical Center/Providence-St.Joseph Health and University of Washington; A.W. Armstrong, MD, MPH, University of Southern California; K. Callis Duffin, MD, University of Utah;

\author{
P.S. Helliwell, DM, PhD, FRCP, University of Leeds, and Bradford \\ Hospitals NHS Foundation Trust; L.J. van Mens, Resident of \\ Rheumatology, Amsterdam University Medical Centers/University of \\ Amsterdam, Department of Clinical Immunology and Rheumatology \\ Amsterdam, Infection \& Immunity Institute; C.T. Ritchlin, MD, \\ MPH, Professor of Medicine, Division of Allergy, Immunology, and \\ Rheumatology, University of Rochester Medical Center; O. FitzGerald, \\ $M D$, FRCPI, FRCP (UK), Newman Clinical Research Professor, \\ Department of Rheumatology, St. Vincent's University Hospital and \\ Conway Institute for Biomolecular Research, University College Dublin. \\ Prof. FitzGerald and Prof. Ritchlin are joint senior authors. \\ Address correspondence to Dr. O. FitzGerald, Newman Clinical \\ Research Professor, Department of Rheumatology, St. Vincent's \\ University Hospital and Conway Institute for Biomolecular Research, \\ University College Dublin, Belfield Dublin 4, Dublin, Ireland. \\ E-mail: oliver.fitzgerald@ucd.ie
}

The Group for Research and Assessment of Psoriasis and Psoriatic Arthritis (GRAPPA)-Collaborative Research Network (CRN) held its third annual meeting at the GRAPPA 2019 annual meeting in Paris, France. The GRAPPA-CRN meeting was organized by a committee that was co-chaired by Professors Oliver FitzGerald and Christopher Ritchlin. The meeting was attended by 29 rheumatologists, 2 dermatologists, 6 patient research partners (PRP), 15 people from the pharmaceutical industry, 2 nonclinical scientists, and an administrator.

Continuing its discussions from the 2018 and 2019 GRAPPA-CRN meetings, the group identified 3 key unmet needs to further the understanding of psoriatic disease: (1) psoriatic arthritis (PsA) Biomarkers for Joint Damage (BioDAM), reflecting the need to identify soluble biomarkers as predictors of structural damage in PsA; (2) PreventPsA, the need to evaluate biomarkers as predictors of the development of PsA among patients with psoriasis; and (3) PredictorPsA, the need to identify biomarkers that predict treat-

Personal non-commercial use only. The Journal of Rheumatology Copyright @ 2020 . All rights reserved. 
ment response in patients with early psoriasis and PsA. The 2019 GRAPPA-CRN meeting's objective was to consolidate components of the infrastructure that are required to progress the identified unmet needs. In particular, the group sought to obtain consensus on (1) the establishment of a shared electronic database and electronic case report form (eCRF); (2) the minimal clinical dataset to be collected; (3) the finalization of standardized operating procedures (SOP) that detail sample collection and storage; and (4) the agreement on the scientific methodology to be used. A multicenter prospective pilot study that addresses PredictorPsA was proposed to test the feasibility of these 4 components. The overall outcome was to develop the framework for obtaining more sustainable funding and to establish goals for a GRAPPA-CRN investigator-initiated multicenter pilot study.

\section{Shared eDatabase and eCRF}

To speed the use of a standardized set of data elements and to help ease the sharing of data across centers, a shared eCRF and database platform for future studies was considered. Robin Waxman, led by Dr. Alexis Ogdie, provided a summary of these findings. A subcommittee that included Waxman and Drs. Ogdie, Vinod Chandran, Oliver FitzGerald, Philip J. Mease, and Denis O'Sullivan was convened to examine the available options. The subcommittee created a criteria list to define the shared platform's requirements. The criteria were then rated by importance as (1) security and compliance; (2) cost; and (3) other factors, including usability, functionality, sustainability, support, standardized dataset, and whether the platform was already "tried and tested" by GRAPPA members in active studies. These criteria were then further discussed and expanded so that a detailed assessment could be undertaken.

The platforms that GRAPPA members currently use are REDCap (Research Electronic Data Capture), DADOS, and OpenClinica. The group conducted a survey at the 2019 GRAPPA-CRN meeting of CRN members and found that 20 centers are currently using REDCap, 5 are using DADOS, and 1 is using OpenClinica. An initial review of these platforms appeared to indicate very little difference between the systems, other than cost.

REDCap. REDCap (www.project-recap.org) is a secure Web application that builds and manages online surveys and databases. It was created at Vanderbilt University in 2004 for a small group of clinical researchers who needed a secure data collection tool that met the US Health Information Portability and Accountability Act compliance standards. The REDCap Consortium states that it has 3763 active partners in 132 countries and that it supports 797,000 projects and 1,082,000 users. REDCapis available at no cost to REDCap Consortium partners. However, some cost would be incurred for trained coordinator support to set up the dataset (this cost would also be incurred for DADOS and OpenClinica). REDCap is not an open-source product.
$D A D O S$. The DADOS platform (www.dadosproject.com) is a Web-based application for data collection in clinical and translational research. DADOS was originally developed for use within various clinics and programs at the University Health Network (UHN) in Canada and is the current platform used by the International Psoriasis and Arthritis Research Team (IPART). The DADOS platform was developed, maintained, and supported by the Electronic Data Capture Program at the Techna Institute. DADOS already has a considerable store of PsA-relevant data, but compared with the other 2 products, is much less widely used. DADOS charges a yearly fee for support and upgrades of about Can\$3500. DADOS is not an open-source product.

OpenClinica. OpenClinica (www.openclinica.com) is an American-based electronic data collection and clinical data management tool. According to the company's Website, this platform supports about 4000 studies spanning over 100 countries with over 5 million participants. OpenClinica is available without charge and has achieved both International Organization for Standardization (ISO) 9001 and ISO 27001 certification. OpenClinica is an open-source product.

Barcoding. Barcoding of samples is deemed essential for integration within a new shared system. REDCap, DADOS, and OpenClinica each support barcoding, but OpenClinica supports both linear and QR barcodes and appears to provide a very useful barcoding system. CRN members reported that barcoding systems such as Freezerworks and LabVantage are available to link patient data to specimen data. Many of these systems also integrate with other data collection platforms. It was reported that, in Iceland, sample barcodes are scanned directly into REDCap through a 2-step process that first labels the specimen and then scans the barcode field in REDCap. The DADOS system in Toronto will also link sample barcodes to patient records, but the key is held by the investigators and not by the system.

\section{Platform review.}

A. Security and compliance issues. All 3 systems were deemed to have high future sustainability. Confidentiality and security compliance were considered good for all 3 systems, but there are currently no European sites using DADOS, so General Data Protection Regulation compliance has not yet been tested. Only OpenClinica has achieved ISO 27001 certification.

B. Data entry issues. All 3 systems allow direct data entry through a Web-based eCRF, allow for local data to be imported from Excel or delimited text, and record the data entry source (as long as variables are encoded exactly as specified). REDCap also allows for double entry and the entry of local data that is sent by fax or that is scanned int o a central location, whereas DADOS does not.

C. Functionality. All 3 systems will (1) support multiple, individual logins through the Internet with simultaneous

Personal non-commercial use only. The Journal of Rheumatology Copyright @ 2020 . All rights reserved. 
data entry and customizable access privileges; (2) record international study data through local research sites, including hospitals; (3) ease data export in a variety of formats, local customized downloads, and reports; and (4) support the collection of patient-reported outcomes (PRO), composite measures, and single-outcome measures. Some REDCap and DADOS users have raised data extraction challenges as a potential issue.

Discussion on shared electronic database. Following a critical review of each platform, including discussion and feedback from CRN members at the 2019 GRAPPA-CRN meeting, it appears that REDCap would best fulfill the needs of the CRN shared data platform. The 20 REDCap users in attendance were enthusiastic about the functionality and user friendliness of the product. Discussion about the 3 products suggests, however, that there are no substantial differences between the platforms. The deciding factor may be the familiarity that GRAPPA members already have with REDCap.

It was also determined that a shared database platform will only be feasible for future studies and not for legacy data. Data currently held by a variety of platforms across different sites and studies could be combined for individual study questions, but because of differing data collection methods and differing variables, this would be difficult and time-consuming.

\section{Minimum Dataset}

Once a shared platform is decided upon, a shared dataset will need to be defined that includes precise question construction and response items for each variable. It is proposed that a minimum dataset be collected in all CRN studies, with additional items added depending on the clinical question being addressed. This would allow for comparisons to be made across studies or for datasets to be combined. To begin the process of defining a shared minimum dataset, the subcommittee created a list of 100 potential core variables under the following categories: demographics, clinical outcome measures, composite measures, PRO measures (PROM), and laboratory measures. All $(\mathrm{n}=70)$ GRAPPA-CRN members, including PRP, were surveyed and asked to identify which items might be included in a minimum dataset for all patients in a Web-based database. There was a $60 \%$ response rate (42/70) to the survey. Out of the 100 items, 27 were judged to be critical by $>70 \%$ of respondents, while 18 were judged to be critical by $50-70 \%$ of respondents (Table 1). Both the items judged as critical by more than $70 \%$ and by $50-70 \%$ will be included in the minimum dataset. The variables that were deemed optional, and those that were rejected by more than $50 \%$ of respondents $(n=56)$, might be included in specific studies but will not be included in the minimal dataset.

Discussion on minimal dataset. The GRAPPA-CRN group provided feedback that the database needed to include more detail on items such as skin history. It also acknowledged more granularity in variable definitions for question construction and response items. In this regard, other groups, such as the GRAPPA-OMERACT group (which is developing outcome measures for the core domain set) must be involved in the process of deciding core $\mathrm{CRN}$ measures given their level of expertise in this area and familiarity with relevant outcome measures. It was also agreed that the eCRF's final composition should be hypothesis driven, and that there needs to be a balance between being comprehensive and being feasible, to optimize site recruitment and retention. Thus, the final set of variables should be agreed upon only once the study questions have been refined.

\section{Biological Samples (Biosample) Collection and Storage SOP}

An SOP is "a document which describes the regularly recurring operations relevant to the quality of the investigation. The purpose of a SOP is to carry out the operations correctly and always in the same manner. A SOP should be available at

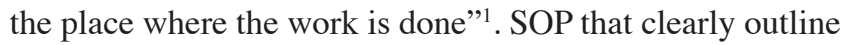
in detail the standard operations related to biosample collection or that are carried out in the laboratory are integral to providing consistent quality, reproducibility, and scientific rigor. It was acknowledged that, for biosamples to be shared with GRAPPA members and for study data to be merged, it is essential to establish agreed SOP for sample collection, storage, shipping, and individual laboratory analysis protocols. Professor Ritchlin provided some background on the importance of scientific rigor and reproducibility. $\mathrm{He}$ stated that $<25 \%$ of high-profile science is reproducible, and that an estimated $85 \%$ of biomedical research is wasted at large ${ }^{2,3}$. Mechanisms that have been adopted to improve reproducibility include the use of (1) US National Institutes of Health training initiatives that ensure grant reviewers promote rigor; (2) journal checklists to blind, randomize, and calculate sample size; and (3) biostatistician reviewers. It is recognized, however, that research institutions should play their part to oversee research integrity (especially those that receive public funding) and that the concept of Good Institutional Practice (GIP) should be adopted ${ }^{2}$. The proposed GIP's pillars include (1) the routine discussion of research methods to critique the methodology used and systematic errors; (2) the use of reporting systems where research integrity can be questioned; (3) the adoption of compulsory institutional training and standards; (4) the use of robust record-keeping and quality management systems; (5) the use of appropriate incentive and evaluation systems; and (6) the enforcement of GIP with penalties for poor-quality research.

Good research practices have been the mission of the University of Rochester Center for Musculoskeletal Diseases, and SOP for assays performed in the laboratory are posted on a publicly available Website (see below), according to Professor Ritchlin. The preclinical and translational

Personal non-commercial use only. The Journal of Rheumatology Copyright $(\subset) 2020$. All rights reserved. 
Table 1. CRN shared database core dataset survey results.

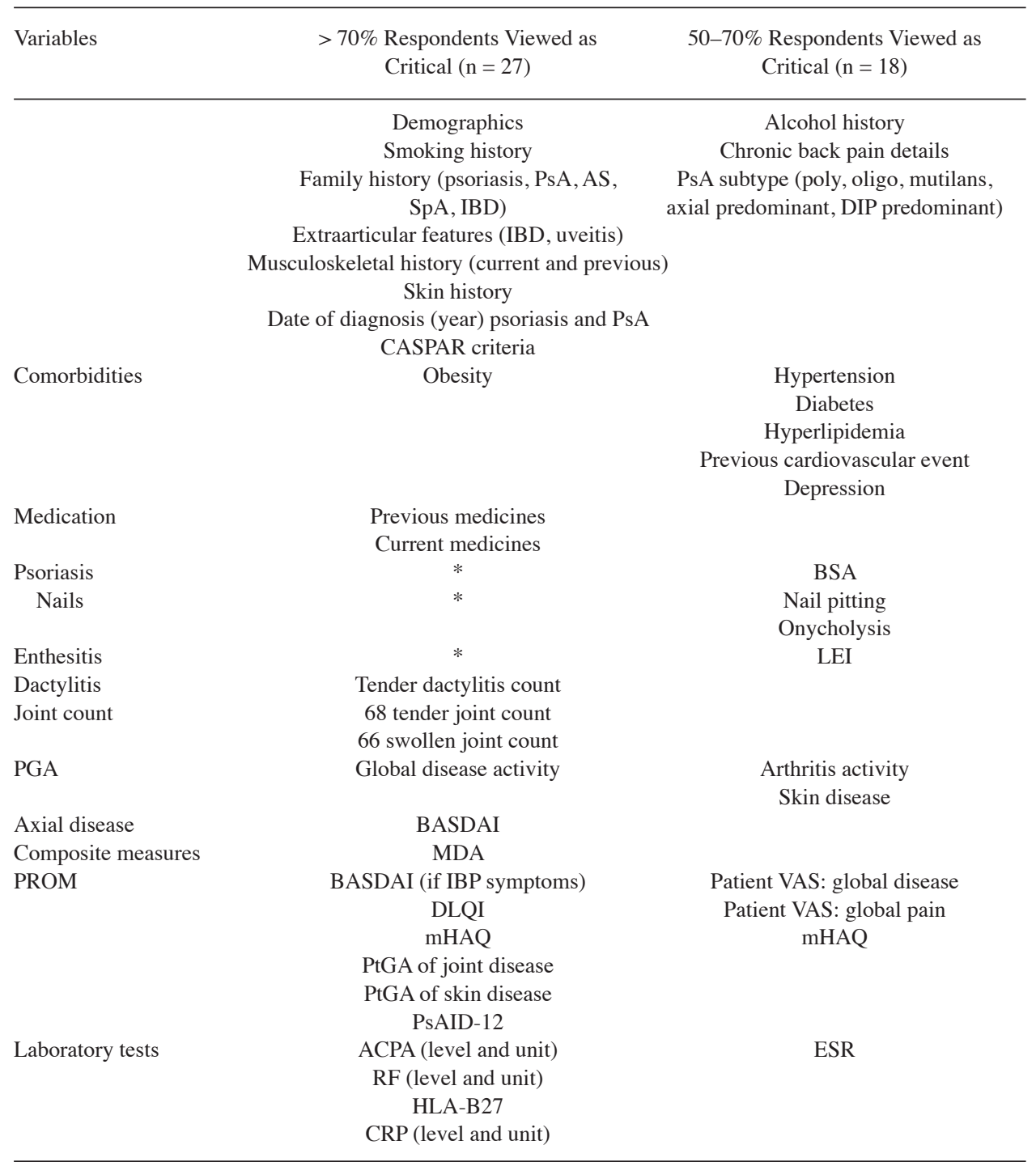

\footnotetext{
$*>70 \%$ respondents agreed that domain items should be included, but there was no consensus on which instrument items to include. CRN: Collaborative Research Network; PsA: psoriatic arthritis; AS: ankylosing spondylitis; SpA: spondyloarthritis; IBD: inflammatory bowel disease; DIP: distal interphalangeal; CASPAR: ClASsification for PsA; BSA: body surface area; LEI: Leeds Enthesitis Index; PGA: physician's global assessment; BASDAI: Bath Ankylosing Spondylitis Disease Activity Index; MDA: minimal disease activity; PROM: patient-reported outcome measures; IBP: inflammatory back pain; VAS: visual analog scale; DLQI: Dermatology Life Quality Index; mHAQ: modified Health Assessment Questionnaire; PtGA: patient's global assessment; PsAID-12: PsA Impact of Disease 12 questionnaire; ACPA: anticitrullinated protein antibodies; ESR: erythrocyte sedimentation rate; CRP: C-reactive protein; RF: rheumatoid factor.
}

research core closely interacts with the data management, bioinformatics, and analytics core to facilitate GIP. Professor Ritchlin presented the "Histology, Biochemistry, and Molecular Imaging (HBMI) Core" within his facility, which includes the mission of the facility, the services provided, and the electronic links to SOP that the facility uses for review by service users (www.urmc.rochester.edu/musculoskeletal-research/core-services/histology/protocols.aspx).

In addition, Professor Ritchlin outlined Quality Re- search Practices that are currently in place within the University of Rochester Center for Musculoskeletal Diseases, which align well with the GIP proposals of Begley, et al and the principles embodied by the Responsible Conduct of Research $(\mathrm{RCR})^{4}$. Professor Ritchlin concluded that the pillars encompassed in GIP and RCR should be applied to research activities and personnel involved in the GRAPPA-CRN.

Carmel Stober reported that the GRAPPA-CRN has previously conducted 2 surveys among the GRAPPA mem- 
bership that identify information about the processing and storage of collected material, as well as what biosamples were being collected; whether ethical approval was in place for the transport of anonymized material; and what personnel were in place ${ }^{5,6}$. Thirty-one international centers provided feedback, with $97 \%$ agreeing to adopt centralized SOP going forward and $81 \%$ preferring that the GRAPPA-CRN develop a standardized template for ethical applications. Regarding biosample collection, $43 \%$ of the centers were storing whole blood for DNA, 33\% were storing whole blood for RNA, and $41 \%$ were storing processed and cryopreserved peripheral blood mononuclear cells (PBMC). A smaller proportion were storing tissue samples, which were mostly snap-frozen or fixed rather than disaggregated for single-cell analysis.

The GRAPPA-CRN SOP subcommittee's pilot work has been to obtain feedback on SOP that members are currently using to compare and contrast methods, and then to draft harmonized SOP for subsequent feedback to the CRN membership. The current focus of the draft harmonized SOP is for (1) biosample collection that can be reproduced in a robust fashion, (2) biosample processing, and (3) subsequent storage methods.

Discussion of SOP for biosamples. Feedback obtained on circulating proposed SOP included the use of standardized collection vessels (e.g., BD Vacutainer CPTTM Cell Preparation tubes for separation of mononuclear cells in a 1-tube system); the use of standardized cryopreservation reagents (e.g., Recovery or CryoStor CS10); and for RNA, the need to adopt technology that applies to multiple cells and tissues to harmonize data across sites. An example presented in the feedback was that different transcriptomic data had been obtained when using either commercially available Applied Biosystems Tempus Blood RNA tubes when compared to Qiagen PAXgene Blood RNA tubes. However, the Accelerating Medicine Partnership (AMP) has provided a platform for moving forward. AMP has taken 2 years to optimize and subsequently validate SOP related to the collection, disaggregation, storage, and downstream processing of synovial tissue ${ }^{7,8}$. Based upon the AMP project's discussions and experience, it is possible that SOP validation will take place across several centers in parallel, and this may result in an application for validation by the US Food and Drug Administration (FDA). One of the key points about qualityassuring synovial biopsies is that samples should be validated by histochemistry prior to performing downstream omics to ensure that the sample includes a synovial lining layer and sublining as a minimum standard.

\section{Investigator-initiated Pilot Study Proposal}

Professor FitzGerald presented a proposal for a GRAPPA-CRN multicenter pilot study that would address PredictorPsA, a key unmet need in PsA where it is proposed to identify biomarkers that predict treatment response in patients with early PsA. This proposed study would also allow researchers to obtain good quality clinical data and associated biosamples to assist in addressing this key area of unmet need. In particular, the study would consider whether there are biomarkers that are identifiable at baseline that will help predict the subsequent treatment response to a targeted therapy.

The proposed pilot study would include 5 centers with a record of clinical research, biosample collection, and storage. Each center would include 8 patients with active psoriasis and PsA requiring treatment intervention according to standard criteria $(n=40)$, who would be followed for 6 months. The primary clinical response endpoint would be the achievement of minimal disease activity (MDA) at 16 weeks, together with a number of secondary clinical response measures. The estimated number of responders (assuming $25 \%$ reach MDA) would be $\mathrm{n}=10$, and nonresponders (assuming a 30\% nonresponse, defined as non-achievement of an ACR20) would be $n=12$. Comparison of various omics results of these extreme cases will be made. The pilot study would evaluate multisite collection of clinical data on an agreed eCRF at baseline, 3, and 6 months. Biosample collection and storage would be undertaken at defined intervals using harmonized SOP, and biosamples would be transported to UHN/IPART for downstream analysis also using agreed SOP.

For the purpose of biosample discovery and validation, it was proposed that liquid samples, including whole blood, could be subjected to several platforms in parallel [discovery; e.g., liquid chromatography-mass spectrometry (MS)/ MS, transcriptomics, bulk flow cytometry], to identify individual or groups of biomarkers that define responders and nonresponders. Identified biomarkers could be subsequently validated in all patients using assays such as multiple reaction monitoring. Tissue samples (synovium and skin) would be taken at baseline and 12 weeks and would be subjected to immunohistochemistry (IHC), disaggregation, and single-cell analysis. For IHC, tissue would be embedded in optimal cutting temperature; included cell surface markers would be $\mathrm{CD} 3, \mathrm{CD} 4, \mathrm{CD} 8, \mathrm{CD} 68$, and vascularity markers (factor VIII); and markers would be quantified using digital image analysis. Tissue disaggregation and single-cell analysis would adopt optimized techniques from the AMP project $^{7,8}$.

Discussion of the pilot project. GRAPPA-CRN meeting attendees recognized that robust SOP for sample collection and storage would need to be in place prior to performing discovery and validation techniques. Attendees felt strongly that the pilot study should address a hypothesis rather than only being a pure feasibility study. They suggested that currently validated biomarkers from other studies could be included (such as baseline matrix metalloprotease 3 and interleukin 6) as markers that predict response to biologic treatment and that the results of studies that use these markers could be used to develop a study hypothesis and

Personal non-commercial use only. The Journal of Rheumatology Copyright (C) 2020. All rights reserved. 
power calculations. Attendees preferred a single biological disease-modifying antirheumatic drug to be used in the first instance. Qualification of SOP could be achieved by working in partnership with the FDA at an early stage. FDA qualification of SOP could also be achieved for biomarkers in addition to clinical outcomes and PROM.

At the 2019 GRAPPA-CRN annual meeting, the CRN group determined that REDCap was the most popular database platform, because it is already being used by 20 GRAPPA centers. The group prioritized eCRF items but determined that the final composition should depend on the individual study and the research questions addressed. The group also agreed that eCRF should only be used for prospectively collected data due to difficulties in combining legacy data. It was decided that feasibility at collection sites needs to be carefully considered, that barcoding needs to be the standard, and that barcoding should be integrated with patient biosamples. In addition, the GRAPPA-CRN should be devising its own GIP strategies and working early with the FDA. A document containing information on SOP used by GRAPPA centers has been collated, but the precise project description will influence the SOP that are selected. Finally, a pilot project that addresses treatment response was presented with plans to discuss the project further with individual pharmaceutical companies.

\section{REFERENCES}

1. Standard Operating Procedures. [Internet. Accessed February 5, 2020.] Available from: www.fao.org/3/w7295e/w7295e 04.htm

2. Begley CG, Buchan AM, Dirnagl U. Robust research: institutions must do their part for reproducibility. Nature 2015;525:25-7.

3. Begley CG, Ioannidis JP. Reproducibility in science: improving the standard for basic and preclinical research. Circ Res 2015; 116:116-26.

4. Resnik DB, Shamoo AE. Reproducibility and research integrity. Account Res 2017;24:116-23.

5. Jadon DR, Chandran V, Ogdie A, Pennington SR, Stober C, Gladman DD, et al. Proceedings of the 2018 GRAPPA Collaborative Research Network Meeting. J Rheumatol Suppl 2019;95:11-9.

6. Jadon DR, Chandran V, Stober C, Ogdie A, Armstrong AW, Callis Duffin K, et al. Proceedings of the 2017 GRAPPA Collaborative Research Network Meeting. J Rheumatol Suppl 2018;94:54-61.

7. Lewis MJ, Barnes MR, Blighe K, Goldmann K, Rana S, Hackney JA, et al. Molecular portraits of early rheumatoid arthritis identify clinical and treatment response phenotypes. Cell Rep 2019; 28:2455-70.

8. Zhang F, Wei K, Slowikowski K, Fonseka CY, Rao DA, Kelly S, et al. Defining inflammatory cell states in rheumatoid arthritis joint synovial tissues by integrating single-cell transcriptomics and mass cytometry. Nat Immunol 2019;20:928-42. 